\title{
Endmember extraction algorithms from hyperspectral images
}

\author{
Pablo J. Martínez, Rosa M. Pérez, Antonio Plaza, Pedro L. Aguilar, María C. Cantero and Javier Plaza \\ Computer Science Department, University of Extremadura, Cáceres, Spain
}

\begin{abstract}
During the last years, several high-resolution sensors have been developed for hyperspectral remote sensing applications. Some of these sensors are already available on space-borne devices. Space-borne sensors are currently acquiring a continual stream of hyperspectral data, and new efficient unsupervised algorithms are required to analyze the great amount of data produced by these instruments. The identification of image endmembers is a crucial task in hyperspectral data exploitation. Once the individual endmembers have been identified, several methods can be used to map their spatial distribution, associations and abundances. This paper reviews the Pixel Purity Index (PPI), $N$-FINDR and Automatic Morphological Endmember Extraction (AMEE) algorithms developed to accomplish the task of finding appropriate image endmembers by applying them to real hyperspectral data. In order to compare the performance of these methods a metric based on the Root Mean Square Error (RMSE) between the estimated and reference abundance maps is used.
\end{abstract}

Key words endmember extraction - hyperspectral mixtures - linear spectral unmixing

\section{Introduction}

Imaging spectroscopy is the measurement and analysis of spectra acquired as images. The use of hyperspectral imaging sensor data to study the Earth's surface is based on the capability of such sensors to provide hundreds of spectral bands (high resolution spectra), providing one spectrum per pixel, along with the image data. This capability can be used to determine Earth's surface constituent signatures from the spectral information provided by the sensor.

The imaging spectroscopy concept can be considered in the general problem of solving unknowns from measurements. Unknowns in-

Mailing address: Dr. Pablo J. Martínez, Computer Science Department, University of Extremadura, Avda. de la Universidad s/n, 10071 Cáceres, Spain; e-mail: pablomar@unex.es clude all the absorbing and scattering components of the atmosphere and the surface that affects the radiance (Green et al., 1998).

If a problem has more unknowns than measurements, then the problem is underdetermined. If there are more measurements than unknowns then the problem is over determined and solvable. The imaging spectroscopy approach leads to over determined, therefore solvable problems.

The problem must be solved taking into account all the unknowns that may affect the expression of interest (there is no magic band for any single material). As the number of equations (bands) increases, the problem is more easily solved.

In most cases, the sub-scene represented by each observed pixel is not homogeneous. As a result, the hyperspectral signature collected by the sensor at each pixel is formed by an integration of signatures, associated with the purest portions of the sub-scene. These signatures, which can be considered macroscopically pure, are usually named «endmembers» in hyperspectral analysis terminology (Bateson et al., 2000). 
In this paper, we review some endmember extraction techniques and use a novel approach to compare the accuracy of these methods.

The rest of the paper is structured as follows: Section 2 provides a description of the concepts of endmember and fully constrained linear spectral unmixing. Section 3 discusses the Pixel Purity Index (PPI), $N$-FINDR and Automatic Morphological Endmember Extraction (AMEE) techniques. Section 4 describes the result achieved after applying the proposed algorithms to a real mineral mapping application, and establishes a comparison between them, using real data collected by the NASA Jet Propulsion Laboratory's Airborne Visible InfraRed Imaging Spectrometer «AVIRIS». Finally, Section 5 includes some concluding statements and comments on plausible future research.

\section{Endmembers in hyperspectral images}

In order to understand the endmember concept, we will analyze the spectrum composition model, considering that a spectrum is an $\mathrm{N}$-dimensional vector.

The radiation source $F$ is characterized by a spectral radiance $f(\lambda)$; this radiation will cross a medium (atmosphere), until it reacts with a sample $i$; this medium will be characterized by a transmittance, $g(\lambda)$ in such a way that the radiation that reaches the sample will be $f(\lambda) g(\lambda)$.

The interaction with the sample will be directed by its spectral reflectance $\rho(\lambda)$; the radi- ation emerging the sample will be, therefore, $f(\lambda) g(\lambda) \rho(\lambda)$ and it will have to suffer a new interaction with the propagation medium, in such a way that the incident radiation over the sensor will be $f(\lambda) \boldsymbol{g}(\lambda) \boldsymbol{\rho}(\lambda) \boldsymbol{g}^{\prime}(\lambda)$, agreeing with spectrometer transfer function $\boldsymbol{h}(\lambda)$, the measurement result, will be

$$
\boldsymbol{x}(\lambda)=f(\lambda) g(\lambda) \rho(\lambda) g^{\prime}(\lambda) \boldsymbol{h}(\lambda) .
$$

The endmember hyperspectral signature $\boldsymbol{\rho}_{i}$, is a reflectance vector obtained from a measurement of a $i$ pure canopy sample $\boldsymbol{x}_{i}$ obtained in the same conditions as the hyperspectral image (detector $h$, source $f$ and atmospheric conditions $g$ and $g^{\prime}$ )

$$
\rho_{i}(\lambda)=\left[\rho_{i, 1}, \rho_{i, 2}, \ldots, \rho_{i, N}\right]^{T} .
$$

Usually the $i$ components present in a non-uniform sample do not participate in this process in the same proportion; if we call $\boldsymbol{\rho}_{i}(\lambda)$ to the $i$ component reflectance spectrum and $\boldsymbol{c}_{\boldsymbol{i}}$ to the abundance of $i$ component in our sample, the measurement process will be like the one shown in fig. 1 .

The composed reflectance spectrum $\rho(\lambda)$ can be expressed as a linear combination of the endmember reflectance signatures $\boldsymbol{\rho}_{i}(\lambda)$ when each isolated source $i$ stimulates the detector separately, $i=1, \ldots, K$, (where $K$ is the number of endmembers). According to the related notation, the superposition principle can be generalized by

$$
\rho=R c=\sum_{i=1}^{K} c_{i} \rho_{i}
$$

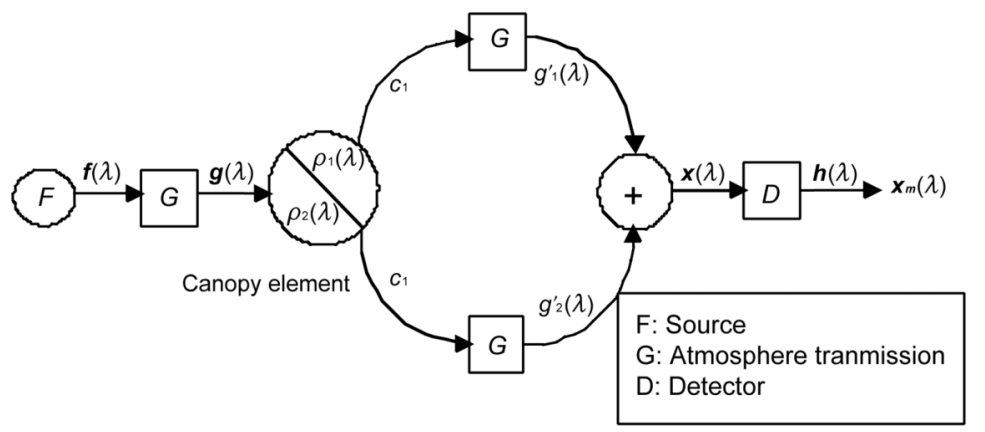

Fig. 1. Spectrum composition. 


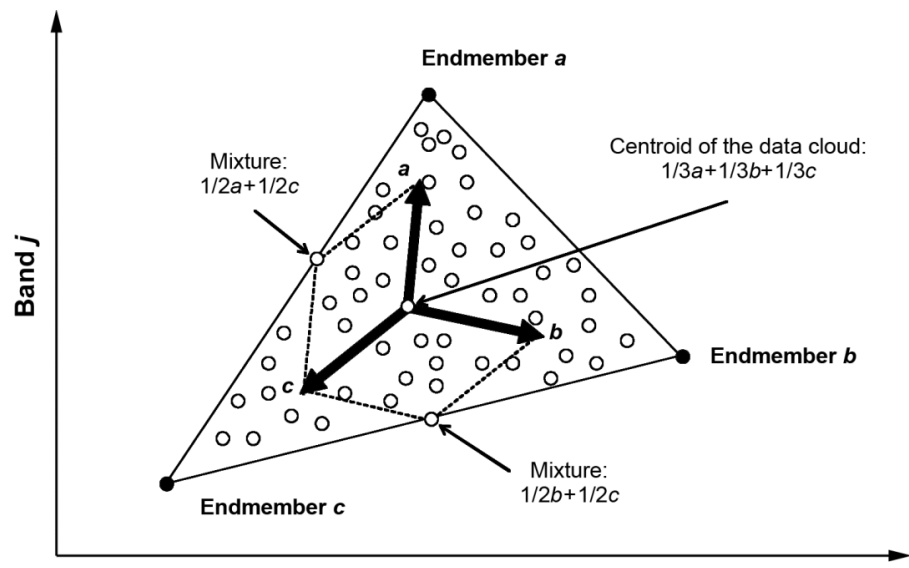

Band $i$

Fig. 2. Mixture model based on three endmembers.

where $R=\left[\rho_{1}, \rho_{2}, \ldots, \rho_{k}\right]$ and $c=\left[c_{1}, c_{2}, \ldots, c_{k}\right]^{T}$. For instance, a simple mixture model based on three endmembers has the geometrical interpretation of a triangle whose vertices are the endmembers. Cover fractions are determined by the position of spectra within the triangle, and they can be considered relative coordinates in a new reference system given by the endmembers, as shown in fig. 2 .

Although the development of effective devices with new and improved technical capabilities stands out, the evolution in the design of algorithms for data processing is not as positive. Many of the techniques currently underway for hyperspectral data analysis and endmember extraction have their origins in classic spectroscopy, and thus focus exclusively on the spectral nature of the data (Plaza et al., 2002). Available analysis procedures do not usually take into account information related to the spatial context, which can be useful to improve the obtained classification (Madhok and Langreb, 1999).

\subsection{Full constraint linear spectral unmixing}

The objective of Linear Spectral Unmixing (LSU) is to obtain the fractions of components in the mixture. Unmixing analysis generally be- gins with a few basic endmembers or components. Endmembers such as green vegetation, soil, non-photosynthetic vegetation, and shade are often used. The spectra of these endmembers may come from laboratory measurements, field measurements or from the imaging spectrometer data set itself.

Each spectrum is then atmospherically corrected and the imaging spectrometer data set is then decomposed into the fractions of the endmembers that best replicate the measured spectrum. The output of LSU analysis is a series of endmember fraction images. These images report the derived distribution of the endmembers over the image.

Two constraints that are often considered on this analysis are: that the fractions must sum to 1.0 and negative fractions are not allowed (FCLSU) (Green et al., 1998).

\section{Background on endmember extraction techniques}

A number of algorithms based on the notion of spectral mixture modeling have been proposed to accomplish the complex task of finding appropriate endmembers for spectral unmixing in multi/hyperspectral data. 


\subsection{PPI}

One of the most successful approaches has been the Pixel Purity Index or PPI (Boardman et al., 1995), which is based on the geometry of convex sets (Ifarraguerri and Chang, 1999). PPI considers spectral pixels as vectors in a $\mathrm{N}$-dimensional space.

A dimensionality reduction is first applied to the original data cube by using the Minimum Noise Fraction. The algorithm proceeds by generating a large number of random $N$-dimensional vectors, also called «skewers» (Theiler et al., 2000), through the dataset. Every data point is projected onto each skewer, along which position it is pointed out. The data points which correspond to extreme values in the direction of a skewer are identified and placed on a list. As more skewers are generated, the list grows, and the number of times a given pixel is placed on this list is also tallied.

The pixels with the highest tallies are considered the purest ones, since a pixel count provides a «pixel purity index». It is important to emphasize that the PPI algorithm does not identify a final list of endmembers. PPI was conceived not as a solution, but as a guide; in fact, the author proposed comparing the pure pixels with target spectra from a library and successively projecting the data to lower dimensional spaces while endmembers were identified. There are several interactive software tools oriented to perform this task, but the supervised nature of such tools leads to the fact that the analysis of a scene usually requires the intervention of a highly trained analyst. Then, interactive solutions may not be effective in situations where large scenes must be analyzed quickly as a matter of routine. In addition, randomness in the selection of skewers has been identified by some authors as a shortcoming of the algorithm.

The original implementation of PPI proposes the use of unitary vectors as skewers in random directions of the $\mathrm{N}$-dimensional space. This implementation may be improved by a careful selection of existing vectors to skew the dataset. Intelligent selection of skewers may result in a more efficient behavior of the algorithm. Some tools based on variations of PPI concepts have been proposed (Theiler et al., 2000).

\subsection{N-FINDR}

The $N$-FINDR method (Winter, 1999) is an automated approach that finds the set of pixels which define the simplex with the maximum volume, potentially inscribed within the dataset. First, a dimensionality reduction of the original image is accomplished by MNF. Next, randomly selected pixels qualify as endmembers, and a trial volume is calculated. In order to refine the initial volume estimation, a trial volume is calculated for every pixel in each endmember position by replacing that endmember and recalculating the volume. If the replacement results in a volume increase, the pixel replaces the endmember. This procedure, which does not require any input parameters, is repeated until there are no replacements of endmembers left. It should be noted that both PPI and $N$-FINDR rely on spectral properties of the data alone, neglecting the information related to the spatial arrangement of pixels in the scene.

The $N$-FINDR method is sensitive to the random selection of an initial set of endmembers. If the initial estimation is appropriate, the algorithm will not require to do many iterations until it reaches the optimum solution. On the contrary, an erroneous initial estimation may lead to a high computational complexity of the algorithm.

On the other hand, the algorithm recalculates the volume of the simplex every time a new pixel is incorporated to the endmember set. This property makes the algorithm quite sensitive to noise.

\section{3. $A M E E$}

Mathematical morphology is the science of shape and structure, based on set-theoretical, topological and geometrical concepts (Serra, 1982, 1993). Morphology was originally defined for binary images and has been extended to the grayscale (Sternberg, 2000) and color (Lambert and Chanussot, 2000) image cases, but it has seldom been used to process multi/hyperspectral imagery.

We propose a new application of mathematical morphology focused on the automated extraction of endmembers from multi-dimension- 
al data. This application is fully described in (Plaza et al., 2002). In addition, we list key advantages in the use of morphology to perform this task:

- A first major point is that the extraction of endmembers is basically a non-linear task.

- Furthermore, morphology allows the introduction of a local-to-global approach in the search of endmembers by using spatial kernels (structure elements in the morphology jargon). These items define local neighbourhoods around each pixel. This type of processing can be applied to the search of convexities or extremities within a data cloud of spectral points and to the use of the spatial correlation between the data.

- As a final main step, morphological operations are implemented by replacing a pixel with a neighbor that satisfies a certain condition. In binary/grayscale morphology, the condition is usually related to the digital value of the pixel, and the two basic morphological operations (dilation and erosion) are respectively based on the replacement of a pixel by the neighbor with the maximum and minimum value. Since an endmember is defined as a spectrally pure pixel that describes several mixed pixels in the scene, extended morphological operations can obviously contribute to the location of suitable pixels that replace others in the scene, according to some desired particularity of the pixel, for example, its spectral purity.

Therefore, we propose a mathematical framework to extend mathematical morphology to the multi-dimensional domain, which results in the definition of a set of spatial/spectral operations that can be used to extract reference spectral signatures.

The dilation and erosion operations have been extended to the grayscale image case (Sternberg, 2000). In grayscale morphology, images are handled as continuous valued sets. Let $f(x, y)$ be a gray level function representing an image, and $K$ another set which comprises a finite number of pixels. This set is usually referred to as a kernel or structuring element in morphology terminology. Erosion and dilation of image $f(x, y)$ by structuring element $K$ are respectively written as

$$
(f \otimes k)(x, y)=\operatorname{Min}_{(s, t) \in k}\{f(x+s, y+t)-k(s, t)\}
$$

$$
(f \otimes k)(x, y)=\operatorname{Max}_{(s, t) \in k}\{f(x-s, y-t)+k(s, t)\}
$$

where $k(s, t)$ denotes the weight associated to the different elements of the kernel. The main computational task in dealing with grayscale morphological operations is the query for local maxima or minima in a local search area around each image pixel. This area is determined by the size and shape of structuring element $K$.

If a dilation operation using $K$ is applied over a grayscale image, then the local effect of the operation is the selection of the brightest pixel in a $3 \times 3$-pixel search area around the target pixel. The constraints imposed on the kernel definition cause all pixels lying within the kernel to be handled equally, i.e. no weight is associated with the pixels according to their position along the kernel, and the pixel with maximum digital value is selected. The previous operation is repeated with all the pixels of the image, leading to a new image (with the same dimensions as the original) where lighter zones are developed concerning the size and shape of the structuring element. In contrast, grayscale erosion has the global effect of shrinking the lighter zones of the image.

\subsubsection{Extending mathematical morphology to multi-spectral images}

In grayscale morphology, the calculation of maximum and minimum gray values relies on the definition of a simple ordering relation given by the digital value of pixels. In the case of multi-spectral datasets, this natural assumption is not straightforward, since there is no natural means for the total ordering of multivariate pixels. Hence, the greatest challenge in the task of extending morphology operations to multispectral images is the definition of a vector ordering relation related to pixel purity that allows us to determine the maximum and minimum elements in any family of $\mathrm{N}$-dimensional vectors (Plaza et al., 2002).

We have checked one possibility that relies on the calculation of the distance between every spectral point in the kernel and the center of the kernel data cloud. Then, a local eccentricity 


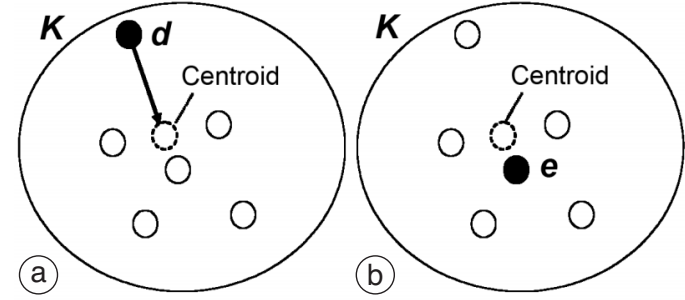

Fig. 3a,b. a) Maximum and b) minimum in data cloud.

measure for a particular $N$-dimensional point of the kernel $\boldsymbol{f}(x, y)$ can be obtained by calculating the distance $\operatorname{dist}(\boldsymbol{f}, \boldsymbol{C})$ in relation to the center $\boldsymbol{C}$ (dist is a point-wise linear distance function spectral angle). Distance $D^{\prime}=\operatorname{dist}(\boldsymbol{f}, \boldsymbol{C})$ defines a partial ordering relation in the data cloud that allows the identification of the maximum as that element which is most «eccentric» or distant from the center (fig. $3 \mathrm{a}$ ), while the minimum is the closest element to the center (fig. 3b).

The result of applying an erosion/dilation operation to a multi-spectral dataset is a new data cube, with exactly the same dimensions as the original, where each pixel has been replaced by the maximum/minimum of the kernel neighborhood considered.

In order to account for the «eccentricity» of the maximum pixel, we propose the combination of the output provided by dilation and erosion operations. While dilation selects the purest pixel (the maximum is the more extremely placed pixel in the data cloud), erosion selects the most highly mixed pixel (the minimum is the pixel that is more exactly placed in the center of the data cloud) in a certain spatial neighborhood. The distance between the maximum and the minimum (a widely used operation in classic mathematical morp $\div$ hology) provides an eccentricity value for the selected pixel that allows an evaluation of the quality of the pixel in terms of its spectral purity. In order to incorporate this idea, a new quality measure must be introduced. We define the Morphological Eccentricity Index (MEI) as the distance between the maximum element (obtained by a dilation) and minimum element (obtained by an erosion) in the kernel.

\subsubsection{Algorithm description}

The Automated Morphological Endmember Extraction (AMEE) method (Plaza et al., 2002) is described as follows: the input to the method is the full data cube, with no previous dimensionality reduction or pre-processing.

Parameter $L$ refers to the number of iterations that are performed by the algorithm. Parameters $S_{\min }$ and $S_{\max }$ denote the minimum and maximum kernel sizes that will be considered in the iterative process, respectively. These parameters are interrelated, as the number of iterations depends on the minimum and maximum kernel sizes under scrutiny.

Firstly, the minimum kernel size $S_{\min }$ is considered. The kernel is moved through all the pixels of the image. The spectrally purest pixel and the spectrally most highly mixed pixel are obtained at each kernel neighborhood by multispectral dilation and erosion operations. A Morphological Eccentricity Index (MEI) value is associated with the purest pixel by comparing the result of the dilation to the result of erosion. The previously described operation is repeated by using structuring elements of progressively increased size, and the algorithm performs as many iterations as needed until the maximum kernel size $S_{\max }$ is achieved.

The associated MEI value of selected pixels at subsequent iterations is updated by means of newly obtained values, as a larger spatial context is considered, and a final MEI image is generated after $L$ iterations.

Once the competitive endmember selection process is finished, endmember extraction is finally accomplished using a spatial-spectral seeded region growing procedure in which the MEI image is used as a reference. Here, a threshold parameter $T$ is used to control the process (Plaza et al., 2002).

\section{Results}

In this section we intend to compare the quality of the endmembers extracted by the PPI, $N$-FINDR and AMEE algorithms when the ground truth information is available and expressed in a set of abundance values. In this ex- 


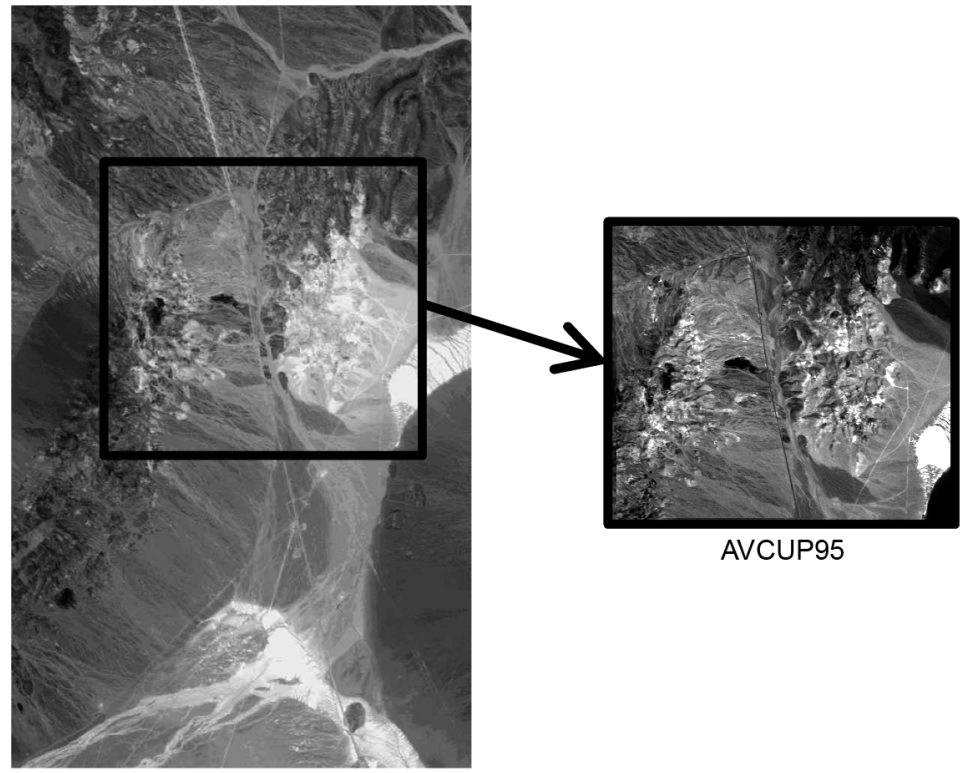

Fig. 4. Location of AVCUP95 image over an aerial photograph of Cuprite mining region, Nevada, U.S.A.

periment we consider that most of the image pixels are a mixture of endmembers, and some of them are pure pixels.

The methodology of comparison is based in the realization of the following steps:

1. The image endmembers are extracted using one of the proposed algorithms.

2. Then, the abundance of each one of the endmembers in the image is estimated, using a FCLSU procedure (Fully Constrained Linear Spectral Unmixing) (Heinz and Chang, 2000). As a result, an abundance map is obtained for each identified spectral signature.

3 . The obtained abundance values are compared, on a pixel-by-pixel basis, with reference values estimated by USGS for the minerals in this area, using the RMSE error. It is important to emphasize that the reference abundances were obtained using USGS Tetracorder algorithm, which produces spectral similarity scores which are consideredin this study as approximated abundance scores. The considered reference maps are available from http://speclab.cr.usgs.gov/.

4. The tests described in this section have been carried out using the image AVCUP95, tak- en by AVIRIS, characterized for having ground truth information in the form of maps that contain the abundance of each mineral in each image pixel.

This image was acquired in 1995 by AVIRIS sensor over the mining region named Cuprite, in the state of Nevada, U.S.A. Figure 4 shows the placement of the image over an aerial photograph of the zone. The scene comprises some areas composed of minerals, as well as abundant bare soils and an interstate road that crosses the zone in a North to South direction.

The image AVCUP95 consists of $400 \times$ $\times 350$ pixels, each one of which contains 50 reflectance values in the range of 2 to $2.5 \mu \mathrm{m}$. This range, placed in the SWIR-II region of the spectrum, is characterized by the manifestation of singularities that allow the discrimination of a wide range of calcareous minerals (Clark, 1999).

Each spectral value in the above mentioned range is equivalent to 10 times the percentage of the reflectance in a given wavelength. This values have been obtained as a result of the application of the ATREM atmospheric correction 
Table I. Global RMSE between the estimated abundances and the real ones for every mineral.

\begin{tabular}{cccc}
\hline \hline Mineral & AMEE & $N$-FINDR & PPI \\
\hline Alunite & 0.05 & 0.05 & 0.05 \\
Buddingtonite & 0.12 & 0.13 & 0.15 \\
Calcite & 0.07 & 0.08 & 0.09 \\
Kaolinite & 0.05 & 0.06 & 0.04 \\
\hline
\end{tabular}

method (Gao et al., 1993), followed by a postprocessing with EFFORT method (Boardman, 1998) over the image in radiance units, originally captured by the sensor, suppressing the effects caused by the atmosphere $g$ and the illumination source $f$.

The ground truth information for the image AVCUP95 has been published by the North American Institute of Geological Studies, or U.S. Geological Survey (USGS from now on). The availability of this information of ground truth, varied and with high quality, favoured AVCUP95 as the image used as a reference to test the performance of new algorithms.

When analysing this image, we centre our study in the most characteristic minerals of the region, that is, alunite, buddingtonite, calcite and kaolinite.

The results regarding the estimation of abundances of alunite mineral are very precise in the three tested methods. The results obtained in the first experiment revealed that the endmember extracted by PPI method for alunite mineral had less spectral similitude with regard to the ground truth than the endmembers extracted by $N$-FINDR and AMEE. However, this fact does not seem to affect the abundances estimation process significantly.

With the aim of establishing a quantitative evaluation of the qualitative results previously mentioned, table I shows the RMSE total errors obtained when comparing each of the estimated abundance maps with their corresponding ground truth map.

The results in table I reveal a very similar behaviour of the three methods, in global terms, for alunite mineral, with an error of $5 \%$ in each of the cases.
Concerning buddingtonite, the calculated error rises in the three cases, being always superior to $10 \%$. This result confirms that there are certain errors in the determination of the spectral shape of the endmember, probably due to atmospheric effects.

Finally, the estimation of the abundance in the case of the calcite and kaolinite minerals is quite precise in all cases, the PPI algorithm being the one that offers the worst results for calcite mineral, with a global error of $9 \%$, and $\mathrm{N}$ FINDR offers the worst results for kaolinite mineral, with a global error of $6 \%$.

The quantitative comparison in table I is useful to obtain a global vision of the precision of the considered methods when it deals with the estimation of abundances.

This study does not reflect the changes caused in the calculated error while the abundance of the component varies. A very interesting question to study in depth is the evaluation of the presented methods in analyzing the magnitude of the calculated error when the material abundance is small and when it is large.

\section{Conclusions}

Spectral unmixing is only possible when the number of endmembers of the image is lower than the number of channels, so that the system of equations to be solved is over-dimensioned; as the system has a larger number of equations we will be able to determine more endmembers when the spectra have more channels.

The best endmembers must be obtained in the same condition as the unmixing spectrum (from the same image) preventing external factors, such as the illumination or elevation angles, affecting the unmixing results.

The endmember extraction methods that jointly exploit spatial and spectral information obtain better quality endmembers than those methods which only utilize spectral information.

It is necessary to design tools for the evaluation of the quality of endmember extraction algorithms using synthetic images designed for that purpose from a previously known abundance map, as well as real images obtained in 
strictly controlled conditions, where it could be possible to obtain the abundance map.

\section{Acknowledgements}

This work was supported by Regional Government Junta of Extremadura by means of the project 2PR03A061. The authors gratefully acknowledge Prof. Pedro Gómez Vilda (Universidad Politécnica de Madrid) for his valuable suggestions and comments on the work described in this paper.

\section{REFERENCES}

Bateson, C.A., G.P. Asner and C.A. Wessman (2000): Endmember bundles: a new approach to incorporating endmember variability into spectral mixture analysis, IEEE Trans. Geosci. Remote Sensing, 38 (2), 10831094.

BOARDMAN, J.W (1998): Post-ATREM polishing of AVIRIS apparent reflectance data using EFFORT: a lesson in accuracy versus precision, in Proceedings of the VII NASA/JPL Airborne Earth Science Workshop.

Boardman, J.W., F.A. Kruse and R.O. Green (1995): Mapping Target Signatures via Partial Unmixing of AVIRIS Data, in Summaries of the V JPL Airborne Earth Science Workshop.

CLARK, R.N. (1999): Spectroscopy of rocks and minerals and principles of spectroscopy, in Principles of Spectroscopy, Manual of Remote Sensing, edited by A.N. RENCZ (John Wiley and Sons, New York), ch. 1, 3-58.

GaO, B.-C., K.B. Heidebrecht and A.F.H. Goetz (1993): Derivation of scaled surface reflectances from AVIRIS data, Remote Sensing Environ., 44, 145-163.
Green, R.O., M.L. Eastwood, C.M. Sarture, T.G. Chrien, M. Aronsson, B.J. ChipPendale, J.A. Faust, B.E. PaVRI, C.J. Choutt, M. Solis, M.R. Olah and O. Williams (1998): Imaging Spectroscopy and the Airborne Visible Infrared Imaging Spectrometer (AVIRIS), Remote Sensing Environ, 65 (3), 227-248.

Heinz, D. and C.-I. ChANG (2000): Fully constrained least squares linear mixture analysis for material quantification in hyperspectral imagery, IEEE Trans. Geosci. Remote Sensing, 39, 529-545.

IfARRAGUERRI, A. and C.-I. ChANG (1999): Multispectral and hyperspectral image analysis with convex cones, IEEE Trans. Geosci. Remote Sensing, 37 (2), 756770 .

LAMBERT, P. and J. CHANUSSOT (2000): Extending mathematical morphology to color image processing, in First Int. Conf. on Color in Graphics and Image Processing (CGIP'2000), October 2000, Saint-Etienne, France, 158-163.

MadHoK, V. and D. LANDGREBe (1999): Spectral-spatial analysis of remote sensing data: an image model and a procedural design, Ph.D. Thesis; and School of Electrical \& Computer Engineering Technical Report TRECE 99-10.

Plaza, A., P. Martínez, R.M. Pérez and J. Plaza (2002): Spatial/spectral endmember extraction by multi-dimensional morphological operations, IEEE Trans. Geosci. Remote Sensing, 40 (9), 2025-2041.

SERRA, J. (1982): Image Analysis and Mathematical Morphology (Academic Press, London).

SERRA, J. (1993): Image Analysis and Mathematical Morphology (Academic Press, London), vol. 1.

SternBerG, S.R. (2000): Greyscale Morphology, Computer Vision Graphics and Image Processing, 35, 283-305.

Theiler, J., D.D. Lavenier, N.R. HARvey, S.J. Perkins and J.J. SZYMANSKI (2000): Using blocks of skewers for faster computation of Pixel Purity Index, SPIE Proc., 4132, 61-71.

WINTER, M.E. (1999): $N$-FINDR: an algorithm for fast autonomous spectral end-member determination in hyperspectral data, SPIE Proc., 3753, 266-275. 